\title{
SimulAção: Plataforma Web de Simuladores Voltados ao Ensino de Física
}

\author{
Fernanda Luíza de Sousa ${ }^{1}$, Silmar Antônio Travain ${ }^{2}$, Guilherme Tavares de Assis ${ }^{1}$ \\ 1- Universidade Federal de Ouro Preto \\ 2- Universidade Estadual Paulista Júlio de Mesquita Filho
}

\begin{abstract}
This article describes the proposal, development, and validation of a simulators' platform focused on the availability and evaluation of computational simulations aimed at the teaching of contents regarding Physics. In its first version, it notably approaches contents on the Conservation of Mechanical Energy. Through practical experiments carried out by a group of users, it was possible to verify a satisfactory usability of the proposed platform and also an adequate understanding of the argumentation process developed by the users when performing the tasks established by the platform.
\end{abstract}

Resumo. Este artigo descreve a proposta, o desenvolvimento e a validação de uma plataforma de simuladores, voltada para a disponibilização e avaliação de simulações computacionais referentes ao ensino de conteúdos de Física; particularmente, em sua primeira versão, conteúdos sobre Conservação da Energia Mecânica. Por meio de experimentos práticos, realizados por um grupo de usuários, verificou-se uma satisfatória usabilidade da plataforma proposta e também uma adequada compreensão do processo de argumentação desenvolvido pelos usuários ao realizar as atividades estabelecidas pela plataforma.

\section{Introdução}

Pesquisas voltadas para o Ensino de Ciências mostram que os alunos possuem melhor desempenho no que se refere à aprendizagem quando participam de forma efetiva das atividades de ensino. No entanto, para que isso ocorra, é essencial uma (re) elaboração dos processos de ensino e aprendizagem que vai desde a utilização de novas metodologias até a mudança de papéis do professor (transmissor) e do aluno (receptor), possibilitando ao aluno a construção do conhecimento e o professor ser mediador desse processo (WILSEK e TOSIN, 2012).

Nas últimas décadas, a evolução da tecnologia faz-se presente nas salas de aulas e na sociedade como um todo, por meio de smartphones, tablets, transmissão de dados por redes sem fio e mídias digitais como programas educacionais, blogs e sites. De acordo com as Orientações Educacionais Complementares aos Parâmetros Curriculares Nacionais (PCNs), a escola deve acompanhar o desenvolvimento da tecnologia e aproveitar os benefícios que esta pode proporcionar (BRASIL, 2002); e, segundo as Notas Estatísticas do Censo Escolar de 2017, dentre as escolas públicas de ensino médio, 79,9\% possuem laboratório de informática (BRASIL, 2016).

Nesse sentido, segundo Araújo e Veit (2004), com os avanços da tecnologia, o professor tem acesso a uma grande quantidade de recursos tais como simulações computacionais, vídeos, animações, entre outros, que podem contribuir para tornar a aula mais interativa e dinâmica, proporcionando ao aluno maior envolvimento no 
VIII Congresso Brasileiro de Informática na Educação (CBIE 2019)

Anais do XXX Simpósio Brasileiro de Informática na Educação (SBIE 2019)

processo de ensino e aprendizagem. Particularmente, estudos mostram que a utilização de simulações computacionais, como recurso didático, pode contribuir para a construção de diferentes representações de uma mesma situação e melhorar a compreensão dos conteúdos, promovendo o desenvolvimento cognitivo, além de proporcionar a observação, problematizar e completar diferentes situações abordadas durante as aulas expositivas (BERTAGNOLLI et al, 2015).

Logo, este trabalho visa o desenvolvimento de uma plataforma Web, denominada SimulAção, para a disponibilização e avaliação de simuladores computacionais voltados para o ensino de conteúdos de Física; particularmente, em sua primeira versão, conteúdos sobre Conservação da Energia Mecânica. Como contribuições deste trabalho, destacam-se: definição de um conjunto de critérios comparativos para ambientes $W e b$ voltados ao ensino de conteúdos quaisquer das áreas de ciências; estabelecimento de um conjunto de simuladores correlacionados e relativos à aprendizagem do tema Conservação da Energia Mecânica; proposta de roteiros de atividades, voltados a cada um dos simuladores; disponibilização da plataforma SimulAção; análise dos argumentos dos alunos usando o modelo de Toulmin; e análise da usabilidade da plataforma SimulAção usando o método do questionário SUS.

Este artigo encontra-se organizado como se segue. A Seção 2 apresenta trabalhos diretamente relacionados ao foco deste trabalho. A Seção 3 apresenta a proposta de desenvolvimento da plataforma SimulAção. A Seção 4 discute a experimentação prática realizada sobre a plataforma e analisa os resultados obtidos. Por fim, a Seção 5 conclui este trabalho e apresenta perspectivas de trabalhos futuros.

\section{Trabalhos Relacionados}

Para a proposta de confecção da plataforma SimulAção, foram analisados os seguintes ambientes $W e b$ :

- Simulações Computacionais de Física (Campos, 2017): consiste em um site offline que visa a disponibilização de roteiros de simulação para aulas práticas no laboratório de informática.

- Currículo de Física e práticas virtuais (Rossi, 2015): consiste em um blog que visa a elaboração e a disponibilização de atividades voltadas para o uso de simulações computacionais com a finalidade de subsidiar professores ao desenvolvimento de suas aulas.

- Simuladores do Movimento Harmônico Simples (MHS) (Carmo, 2017): consiste em uma proposta de disponibilização de simuladores virtuais para o ensino do Movimento Harmônico Simples, a partir do software dinâmico GeoGebra.

- WebQui (Estevão, 2019): consiste em uma plataforma para ensinar Química de forma interativa, dinâmica e visual, possibilitando acesso a conteúdos atualizados e um espaço para troca de experiências, e contribuindo com os professores na dinamização das aulas de Química.

- Simulação Computacional usando o software Modellus (Oliveira, 2014): consiste em um site que objetiva divulgar a modelagem e as simulações criadas para o ensino de energia mecânica e sua conservação, por meio do uso do software Modellus. 
VIII Congresso Brasileiro de Informática na Educação (CBIE 2019)

Anais do XXX Simpósio Brasileiro de Informática na Educação (SBIE 2019)

Visando a proposta da plataforma SimulAção, com base nos trabalhos relacionados apresentados, foram definidos um conjunto de critérios para análise de ambientes $W e b$ voltados ao ensino, a saber:

- Página inicial: visão geral do ambiente $W e b$;

- Apresentação: objetivo do ambiente Web e descrição breve dos autores;

- Roteiros de aula: roteiros para direcionar os alunos quanto às atividades a serem realizadas;

- Link dos simuladores: acesso aos simuladores, de forma rápida e eficiente;

- Ficha técnica: informações técnicas sobre o ambiente Web como, por exemplo, público-alvo, tema e componente curricular;

- Avaliação: processo de avaliação do ambiente $W e b$ a ser realizado pelos usuários do mesmo;

- Contato: possibilidade de envio de comentários, sugestões e/ou propostas de atividade.

A Tabela 1 apresenta, de forma resumida, uma análise comparativa de tais critérios verificados em cada trabalho relacionado.

Tabela 1. Síntese dos critérios verificados em cada trabalho relacionado.

\begin{tabular}{|c|c|c|c|c|c|c|c|}
\hline \multirow{2}{*}{$\begin{array}{c}\text { Trabalho } \\
\text { Relacionado }\end{array}$} & \multicolumn{7}{|c|}{ Critérios analisados } \\
\hline & $\begin{array}{l}\text { Página } \\
\text { inicial }\end{array}$ & Apresentação & $\begin{array}{c}\text { Roteiros } \\
\text { de aula }\end{array}$ & $\begin{array}{c}\text { Link dos } \\
\text { simuladores }\end{array}$ & $\begin{array}{l}\text { Ficha } \\
\text { técnica }\end{array}$ & Avaliação & Contato \\
\hline $\begin{array}{c}\text { Simulações } \\
\text { Computacionais } \\
\text { de Física }\end{array}$ & $\checkmark$ & $\checkmark$ & $\checkmark$ & $\checkmark$ & $x$ & $x$ & $\checkmark$ \\
\hline $\begin{array}{c}\text { Currículo de } \\
\text { Física e Práticas } \\
\text { Virtuais }\end{array}$ & $\mathbf{x}$ & $x$ & $\checkmark$ & $x$ & $x$ & $x$ & $x$ \\
\hline $\begin{array}{l}\text { Simulações do } \\
\text { MHS }\end{array}$ & $\checkmark$ & $\checkmark$ & $\checkmark$ & $\checkmark$ & $x$ & $x$ & $\checkmark$ \\
\hline WebQui & $\checkmark$ & $\checkmark$ & $\checkmark$ & $\checkmark$ & $x$ & $x$ & $\checkmark$ \\
\hline $\begin{array}{c}\text { Modelagem e } \\
\text { Simulação } \\
\text { Computacional } \\
\text { no Ensino de } \\
\text { Física } \\
\end{array}$ & $\checkmark$ & $\checkmark$ & $\checkmark$ & $x$ & $x$ & $x$ & $\checkmark$ \\
\hline $\begin{array}{l}\text { Nova proposta: } \\
\text { SimulAção }\end{array}$ & $\checkmark$ & $\checkmark$ & $\checkmark$ & $\checkmark$ & $\checkmark$ & $\checkmark$ & $\checkmark$ \\
\hline
\end{tabular}

Baseando-se na Tabela 1, diferentemente dos trabalhos relacionados, a proposta de concepção da plataforma SimulAção cumpre, conjuntamente, todos os critérios definidos, sendo o mais completo possível.

\section{Desenvolvimento}

A plataforma SimulAção consiste em uma ferramenta para auxiliar professores, durante as aulas de Física, na utilização de metodologias inovadoras que podem favorecer o ensino e a aprendizagem. Em sua primeira versão, o público alvo da plataforma corresponde a alunos do $1^{\circ}$ ano do ensino médio. 
VIII Congresso Brasileiro de Informática na Educação (CBIE 2019)

Anais do XXX Simpósio Brasileiro de Informática na Educação (SBIE 2019)

Para selecionar a área da Física inicialmente abordada pela plataforma SimulAção, foi realizado um levantamento da quantidade de simuladores públicos e disponíveis, a partir de três principais páginas da Web que disponibilizam simuladores, nas seguintes áreas da Física: movimento; trabalho, energia e potência; calor e termometria; som e ondas/luz e radiação; eletromagnetismo; e física moderna. A Tabela 2 apresenta o resultado do levantamento feito.

Tabela 2: Quantidade de simuladores por área.

\begin{tabular}{|c|c|c|c|c|c|c|}
\cline { 2 - 7 } \multicolumn{1}{c|}{} & Movimento & $\begin{array}{c}\text { Trabalho, } \\
\text { Energia e Potência }\end{array}$ & $\begin{array}{c}\text { Calor e } \\
\text { Termometria }\end{array}$ & $\begin{array}{c}\text { Som e Ondas/ } \\
\text { Luz e Radiação }\end{array}$ & Eletromagnetismo & $\begin{array}{c}\text { Física } \\
\text { Moderna }\end{array}$ \\
\hline $\begin{array}{c}\text { PhET } \\
\text { Interactive } \\
\text { Simulations }\end{array}$ & 29 & 14 & 14 & 31 & 24 & 20 \\
\hline LabVirt & 53 & 17 & 23 & 9 & 16 & - \\
\hline $\begin{array}{c}\text { Applets Java de } \\
\text { Física }\end{array}$ & 13 & 4 & 1 & 10 & 8 & 5 \\
\hline
\end{tabular}

De acordo com a Tabela 2, optou-se por escolher as áreas Movimento e Trabalho, Energia e Potência no intuito de promover o estudo da conservação da energia mecânica, pelo fato de apresentar muitos simuladores disponíveis para uso público; ademais, tais áreas são abordadas no $1^{\circ}$ ano do ensino médio, possuem muitos conceitos abstratos e são difíceis de serem abordadas em um laboratório didático de ciências por meio de materiais alternativos ${ }^{1}$. A partir de tais áreas definidas para a primeira versão da plataforma SimulAção, foram escolhidos três simuladores a serem tratados pela plataforma, que possibilitam o estudo das áreas definidas de forma a representar uma sequência de ensino. A arquitetura de funcionamento da plataforma SimulAção está ilustrada na Figura 1.

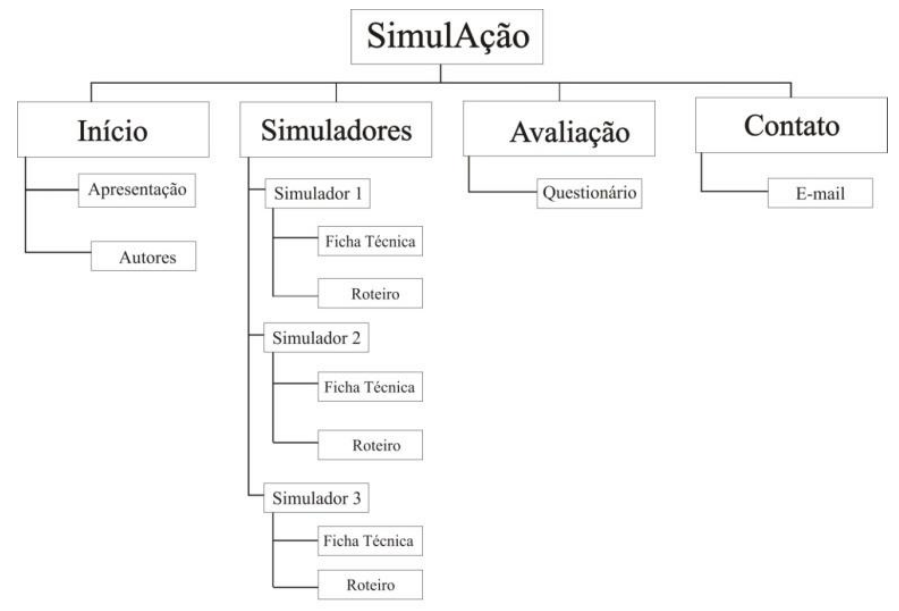

Figura 1. Arquitetura de funcionamento da SimulAção

De acordo com a Figura 1, observa-se que a arquitetura da SimulAção é composta por 4 eixos principais: Início, Simuladores, Avaliação e Contato. O eixo Início apresenta o objetivo da plataforma e os autores da mesma. O eixo Simuladores apresenta, para cada simulador pertencente à plataforma, o próprio simulador, sua ficha

${ }^{1}$ Materiais alternativos são materiais de baixo custo e facilmente acessíveis, encontrados em supermercados e em casas, podendo substituir materiais tradicionais usados em um laboratório. 
VIII Congresso Brasileiro de Informática na Educação (CBIE 2019)

Anais do XXX Simpósio Brasileiro de Informática na Educação (SBIE 2019)

técnica e o roteiro elaborado para utilização do mesmo. $\mathrm{O}$ eixo Avaliação promove a avaliação dos serviços prestados pela plataforma, por meio de um questionário de satisfação subjetiva do usuário. Por fim, o eixo Contato possibilita aos usuários da plataforma o envio de sugestões, comentários e propostas de atividades, via e-mail.

A Figura 2 ilustra a página inicial da plataforma SimulAção que se encontra disponível em https://sitesimulacao.wixsite.com/simulacaoambienteweb. Como se pode observar, logo após da logomarca da plataforma, apresenta-se o menu principal da mesma, sendo composto pelos 4 eixos já descritos na Figura 1. Observa-se também textos introduzindo a plataforma e descrevendo seus autores.

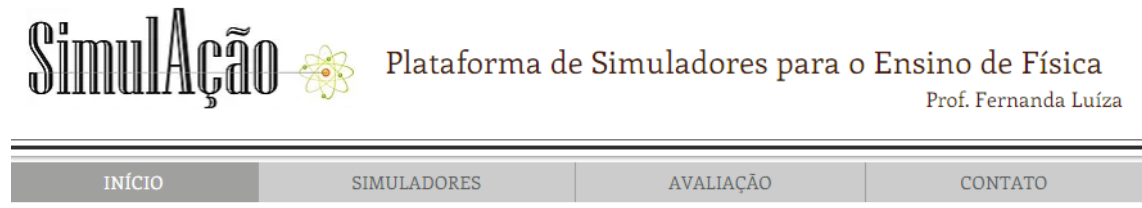

SOBRE

Este site é resultado do produto educacional criado no Programa de Pós-graduação da Universidade Federal de Ouro Preto-MG, do Mestrado Profissional em Ensino de Ciências. Teve como objetivo desenvolver uma plataforma de simuladores para a disponibilização e a avaliação de simuladores computacionais voltados para o ensino de conteúdos da Mecânica.

A SimulAção é uma plataforma de simuladores criada com intuito de auxiliar os professores de Física, proporcionando acesso a conteúdos na área da mecânica, mais especificamente Conservação da Energia, para alunos do $1^{\circ}$ ano do ensino médio. Os simuladores são de domínio público e os roteiros de autoria própria.

Esperamos que essa plataforma seja uma ferramenta útil para os professores que buscam utilizar metodologias inovadoras durante as aulas de Física.

AUTORES

Figura 2. Tela inicial da plataforma SimulAção

Os simuladores, pertencentes à primeira versão da plataforma, são:

- A Rampa ${ }^{2}$ : possui, como objetivos, explicar o movimento de um objeto em um plano inclinado, possibilitando desenhar diagramas de corpo livre, e calcular a força resultante sobre um objeto em um declive.

- A Montanha-russa3 ${ }^{3}$ possui, como objetivos, reconhecer variáveis que caracterizam a energia mecânica no movimento, verificar a conservação de energia cinética e identificar o trabalho da força gravitacional na transformação de energia potencial gravitacional em energia cinética.

- A Pista de Skate ${ }^{4}$ : possui, como objetivo, explicar o conceito de conservação de energia mecânica usando energia cinética e potencial gravitacional, sendo possível explorar diferentes tipos de pistas e visualizar as taxas de energia cinética, energia potencial e a dissipação de energia térmica através do atrito.

\footnotetext{
${ }^{2}$ Disponível em https://phet.colorado.edu/pt_BR/simulation/legacy/the-ramp, acesso em jul. 2019 ${ }^{3}$ Disponível em

http://www.educacional.com.br/Recursos/ConteudoMultimidia/scorm/06_080/06/01/Principal.htm, acesso em jul. 2019.

${ }^{4}$ Disponível em https://phet.colorado.edu/sims/html/energy-skate-park-basics/latest/energy-skate-parkbasics_pt_BR.html, acesso em jul. 2019.
} 
VIII Congresso Brasileiro de Informática na Educação (CBIE 2019)

Anais do XXX Simpósio Brasileiro de Informática na Educação (SBIE 2019)

\section{Análise Experimental}

Nesta seção, é descrita a experimentação prática realizada para avaliar o funcionamento e a usabilidade da plataforma SimulAção (vide Subseção 4.1) e são apresentados e analisados os resultados obtidos (vide Subseção 4.2).

\subsection{Descrição da experimentação prática}

Com o objetivo de verificar os resultados gerados pelo uso de simuladores, quanto ao ensino e aprendizagem dos conteúdos de Física estabelecidos para a plataforma SimulAção e também quanto ao uso geral da própria plataforma, referente ao seu funcionamento e à sua usabilidade, foi realizada uma experimentação por meio da utilização prática de tal plataforma por um grupo de 30 usuários, denominados nãoespecialistas, composto por alunos do $1^{\circ}$ ano do Ensino Médio. Visando novos resultados, futuramente, será realizada uma experimentação envolvendo um grupo de usuários especialistas, sendo composto por professores de Física e alunos do $7^{\circ}$ e $8^{\circ}$ período de cursos de Licenciatura em Física.

A experimentação ocorreu entre os dias 08/05/2019 e 22/05/2019, sendo realizada em encontros com usuários não-especialistas: uso da plataforma SimulAção, envolvendo o roteiro proposto de atividades para cada simulador, e aplicação do questionário SUS, proposto por Brooke (1996), aos alunos visando avaliação do funcionamento da plataforma. O questionário SUS visa avaliar a usabilidade de diversos produtos e serviços como websites, sistemas de informação e produtos de hardware, sendo de aplicação rápida e possuindo alto grau de confiabilidade. É composto, simplesmente, por 10 questões com 5 opções de respostas, em uma escala de 1 a 5 , onde 1 significa "discordo totalmente" e 5 significa "concordo totalmente". Para as questões ímpares, a pontuação da questão equivale à escala (posição) da resposta selecionada pelo usuário menos 1. Já para as questões pares, a pontuação da questão equivale a 5 menos a escala (posição) da resposta selecionada pelo usuário. Para obter a pontuação final, deve-se somar as pontuações resultantes de cada questão e multiplicar tal soma por 2,5, gerando assim o valor do nível de usabilidade. Vale mencionar, que as questões de identificação ímpar referem-se a pontos positivos e as de identificação par referem-se a pontos negativos sobre o uso do produto ou serviço avaliado.

Todos os encontros ocorreram no laboratório de informática de uma escola da rede estadual de ensino do interior de Minas Gerais, que possui 30 computadores em perfeito estado de conservação; entretanto, apenas 10 possuíam acesso a internet, fazendo com que os usuários formassem grupos de 3 para a realização das atividades.

Além dos dados obtidos pelo questionário SUS, para análise dos registros escritos obtidos a partir dos roteiros dos simuladores, foi utilizado o método de Toulmin (1958) que consiste numa ferramenta de análise muito usada para investigar a "argumentação científica" construída por alunos em situações do ensino de Ciências. Para tanto, os textos e as falas dos usuários foram reestruturados com base nos elementos propostos por este método.

\subsection{Análise dos Resultados Obtidos}

No primeiro momento, foram analisadas as respostas dos usuários quanto às questões do questionário SUS utilizado na experimentação. 
VIII Congresso Brasileiro de Informática na Educação (CBIE 2019)

Anais do XXX Simpósio Brasileiro de Informática na Educação (SBIE 2019)

A Figura 3 apresenta, a partir dos valores relacionados às respostas escolhidas pelos usuários para as 10 questões do questionário SUS, as pontuações finais (SUS Score) geradas para os mesmos; ademais, a partir de todas as pontuações finais geradas, apresenta a pontuação geral, relativa à metodologia proposta pelo questionário SUS, para a plataforma SimulAção.

\begin{tabular}{|c|c|c|c|c|c|c|c|c|c|c|c|c|c|c|c|c|c|c|c|c|c|c|c|c|c|c|c|c|c|c|}
\hline Alunos & $\begin{array}{l}\mathbf{A} \\
\mathbf{1}\end{array}$ & $\underset{2}{\mathbf{A}}$ & $\begin{array}{l}\mathbf{A} \\
3\end{array}$ & $\begin{array}{l}\mathrm{A} \\
4\end{array}$ & $\begin{array}{l}\mathbf{A} \\
\end{array}$ & $\begin{array}{l}\mathbf{A} \\
6\end{array}$ & $\begin{array}{l}\mathbf{A} \\
7\end{array}$ & $\begin{array}{l}\mathbf{A} \\
8\end{array}$ & \begin{tabular}{|l|l} 
\\
\end{tabular} & $\begin{array}{c}\text { A } \\
10 \\
\end{array}$ & $\begin{array}{c}\text { A } \\
11\end{array}$ & $\begin{array}{c}A \\
12\end{array}$ & $\begin{array}{c}\mathbf{A} \\
13\end{array}$ & $\begin{array}{c}\text { A } \\
14\end{array}$ & $\begin{array}{c}A \\
15\end{array}$ & $\begin{array}{c}\text { A } \\
16\end{array}$ & $\begin{array}{l}\text { A } \\
17\end{array}$ & $\begin{array}{l}\text { A } \\
18\end{array}$ & \begin{tabular}{|c|c|} 
\\
19
\end{tabular} & $\begin{array}{c}\mathbf{A} \\
20\end{array}$ & \begin{tabular}{|c|c} 
\\
21 \\
\end{tabular} & $\begin{array}{c}A \\
22\end{array}$ & $\begin{array}{l}\mathbf{A} \\
23\end{array}$ & $\begin{array}{l}\mathbf{A} \\
\mathbf{2 4}\end{array}$ & $\begin{array}{c}\mathbf{A} \\
25\end{array}$ & $\begin{array}{c}\text { A } \\
26\end{array}$ & $\begin{array}{c}\mathbf{A} \\
27\end{array}$ & \begin{tabular}{|c}
$\mathbf{A}$ \\
$\mathbf{2 8}$
\end{tabular} & $\begin{array}{c}\text { A } \\
29\end{array}$ & $\begin{array}{c}\mathbf{A} \\
\mathbf{3 0}\end{array}$ \\
\hline $\begin{array}{c}\text { SUS } \\
\text { Scor }\end{array}$ & 72,5 & 80 & 85 & 100 & 95 & 87,5 & 95 & 85 & 85 & 95 & 85 & 100 & 97,5 & 95 & 80 & 62,5 & 77,5 & 100 & 85 & 100 & 87,5 & 97,5 & 90 & 95 & 92,5 & 87,5 & 92,5 & 85 & 85 & 97,5 \\
\hline
\end{tabular}

\begin{tabular}{|l|l|}
\hline Pontuação Geral & 89,08 \\
\hline
\end{tabular}

Figura 3. Pontuações finais SUS para os usuários

De acordo com a Figura 3, a pontuação geral obtida, quanto ao uso da plataforma SimulAção por usuários não-especialistas nesta primeira experimentação, foi de 89,08 que representa, segundo a classificação de Bangor, Kortum e Miller (2009), que a usabilidade da plataforma é aceitável, quanto às faixas de aceitabilidade, e que se encontra entre o excelente e o melhor imaginável, quanto às classificações adjetivas.

\section{Gráfico 1: Porcentagens a favor e contra, para cada questão do questionário SUS}

1. Gostaria de usar o site de simuladores com frequência

2. Utilizar o site de simuladores poderia ser uma tarefa mais fácil.

3. Utilizar o site de simuladores é uma tarefa fácil.

4. Seria necessário um apoio técnico para eu estar apto a usar o site de simuladores.

5 Fiquei satisfeito com os serviços oferecidos pelo site de simuladores.

6. Considero que o site de simuladores apresenta muitas inconsistências em seu.

7. Considero que a maioria das pessoas pode aprender a usar o site de simuladores...

8. Considero que o site de simuladores é muito complicado de utilizar.

9. Enquanto estava utilizando o site de simuladores, senti muito confiante em.

10. Precisaria aprender várias coisas novas para conseguir utilizar o site de simuladores.
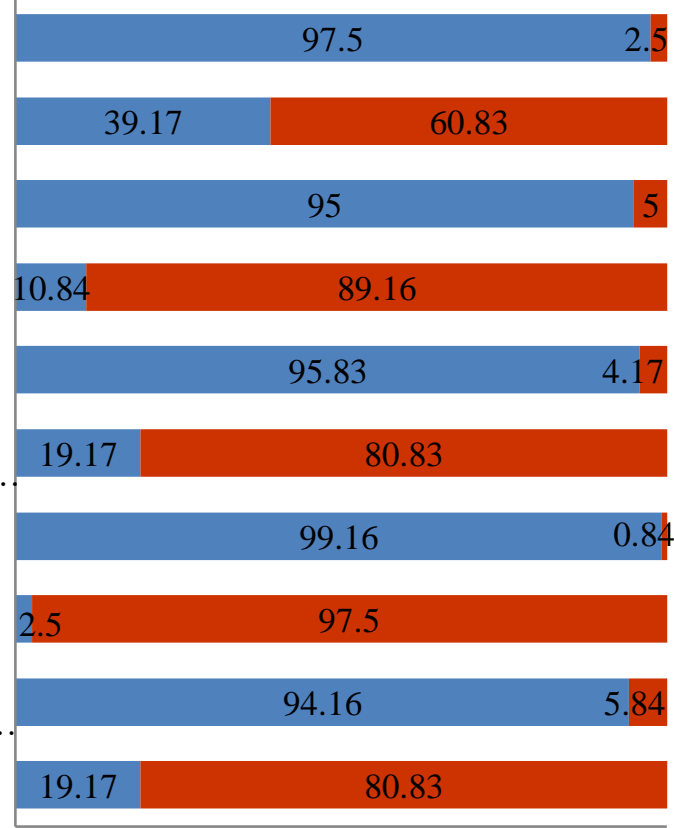

$\begin{array}{lllllllllll}0 & 10 & 20 & 30 & 40 & 50 & 60 & 70 & 80 & 90 & 100\end{array}$

- Porcentagem a favor da afirmação questão

- Porcentagem contra a afirmação da questão

Complementando a análise, o Gráfico 1 apresenta, para cada questão do Questionário SUS, baseando-se nas respostas escolhidas pelos usuários, a porcentagem a favor e contra a afirmação da questão. Considerando a média de 70 pontos como satisfatória para uma determinada questão, de acordo com os estudos do autor citado 
VIII Congresso Brasileiro de Informática na Educação (CBIE 2019)

Anais do XXX Simpósio Brasileiro de Informática na Educação (SBIE 2019)

anteriormente, pode-se observar que apenas a questão 2 não atingiu esta média, o que implica que a execução da plataforma SimulAção, de uma forma geral, pode ter sido um pouco complicada para os usuários não-especialistas, já que estes não estão habituados com a utilização desse tipo de ferramenta. Em contrapartida, a pontuação relativa à questão 3 mostra que tais usuários não tiveram dificuldade em usar a plataforma.

Para exemplificar a utilização do modelo de Toulmin, a Figura 4 apresenta as transcrições obtidas a partir da atividade 1 do simulador A Rampa, aplicada aos alunos.

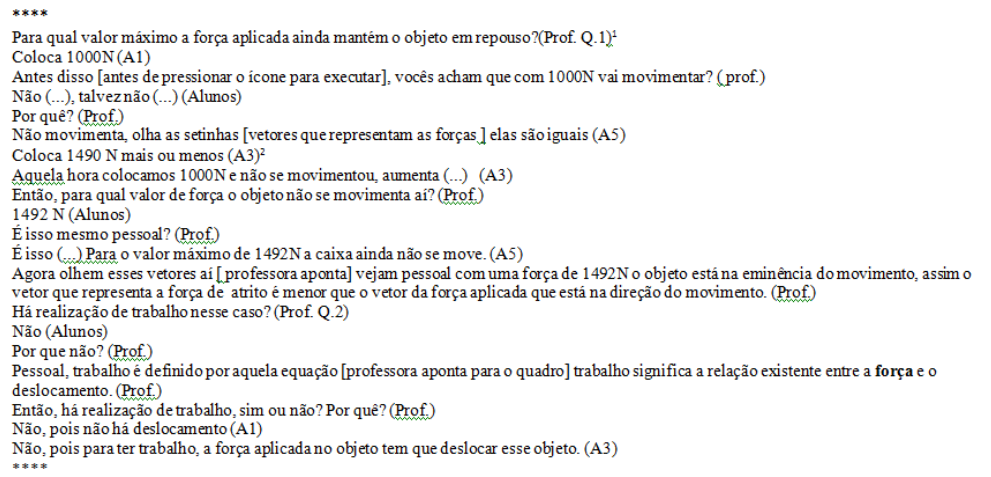

Figura 4. Transcrições referentes à atividade 1 do simulador $A$ Rampa

De acordo com a proposta do modelo de Toulmin, durante a análise, procurou-se evidenciar nas falas dos alunos os processos argumentativos, e pôde-se observar as intervenções do professor, procurando mediar a participação dos alunos para a resolução das questões e para a proposta de novas situações. A Figura 5 apresenta a análise baseada no modelo de Toulmin a partir da transcrição ilustrada na Figura 4.

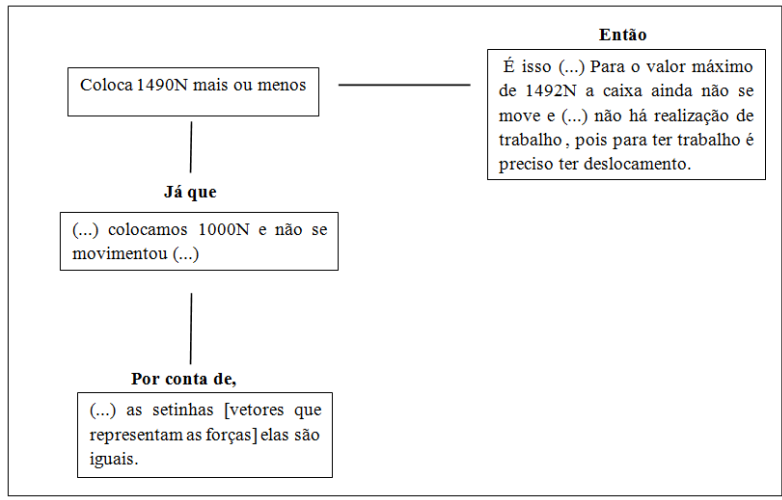

Figura 5. Análise baseada no modelo de Toulmin para a transcrição da Figura 4

De acordo com a Figura 5, pode-se observar que um aluno ao falar "coloca 1490N mais ou menos", ele apresenta sua hipótese. Quando outro aluno diz "(...) as setinhas [vetores que representam as forças] elas são iguais”, ele aponta uma sustentação para a hipótese. Desta forma, relacionando a hipótese, o aluno busca a construção da conclusão: 'É isso (...) Para o valor máximo de $1492 \mathrm{~N}$ a caixa ainda não se move e (...) não há realização de trabalho, pois para ter trabalho é preciso ter deslocamento", construindo assim os primeiros argumentos. Nesta etapa, ocorre o processo de interação por meio da argumentação desenvolvida entre os alunos e o professor, ou seja, a discussão provocada durante a interlocução do professor pode favorecer o desenvolvimento da aprendizagem. A partir dessa interação, pode ocorrer a troca de conhecimentos, uma vez que, durante os encontros de experimentação, a 
VIII Congresso Brasileiro de Informática na Educação (CBIE 2019)

Anais do XXX Simpósio Brasileiro de Informática na Educação (SBIE 2019)

professora ocupava-se em fazer o papel de mediadora, seguindo o que propõe a teoria de Vigotsky (1989) para a situação de estímulo da aprendizagem. Os alunos foram orientados por meio da mediação e interação dos sujeitos (alunos) com os simuladores, nesse caso, objeto de conhecimento. À medida que as dificuldades eram encontradas, os alunos recebiam as orientações da professora; assim, em muitos casos, buscavam juntos os acertos. A orientação compunha-se por perguntas, sugestões e acompanhamentos que, em conjunto, buscavam instigar a argumentação levando os alunos a responderem aos próprios questionamentos.

Dessa forma, de acordo com as Figuras 4 e 5, pode-se verificar que o uso do modelo padrão de Toulmin mostrou-se útil na análise do processo argumentativo enunciado pelos alunos na resolução dos questionamentos.

\section{Conclusão}

A partir da experimentação prática de validação da plataforma SimulAção, realizada com um grupo de usuários não-especialistas, foi possível verificar que a plataforma SimulAção, de acordo com o método do questionário SUS, apresentou uma pontuação geral igual a 89,08, indicando que a usabilidade da plataforma e seus serviços, até o momento oferecidos, é aceitável, encontrando-se entre o excelente e o melhor imaginável; ademais, ainda de acordo com o método do questionário SUS, a plataforma atendeu satisfatoriamente os critérios eficiência, satisfação, facilidade de aprendizagem, facilidade de memorização e minimização de erros. Quanto ao modelo de Toulmin, a partir da análise referente à experimentação prática sobre um dos simuladores, foi possível compreender o processo de argumentação desenvolvido pelos alunos durante a realização das atividades.

Como perspectivas de trabalho futuro, tem-se: a) experimentação prática de validação da plataforma SimulAção com um grupo de usuários especialistas; b) análise de resultados, quanto à utilização do Questionário SUS e do modelo de Toulmin, para a nova experimentação prática; e c) aplicação de técnicas de mineração de dados e/ou aprendizado de máquina nos dados experimentais obtidos, no intuito de encontrar padrões significativos.

\section{Referências}

ARAÚJO, I.S.; VEIT, E.A. "Uma revisão da literatura sobre estudos relativos a tecnologias computacionais no ensino de Física". Revista Brasileira de Educação em Ciências. v.4, n.3, p.5-18, 2004.

BANGOR, A.; KORTUM P. and MILLER J.. Determining What Individual SUS Scores Mean: Adding an Adjective Rating Scale In: Journal of Usability Studies, Vol. 4, Issue 3, May 2009, pp. 114-123.

BERTAGNOLLI, C. M.; BATISTTEL, O. L.; HOLZ, S. M.; STIEGELMEIR, S. H.. "A modelagem e a simulação computacional, como recurso didático na disciplina de Física em cursos técnicos integrados ao ensino médio". Revista Gestão Universitária (Online),V. 5, 2015.

BRASIL. Ministério da Educação. Secretaria da Educação Média e Tecnológica. "Parâmetros Curriculares Nacionais+ $(\mathrm{PCN}+)$ - Ciências da Natureza e suas Tecnologias". Brasília: MEC, 2002. 
VIII Congresso Brasileiro de Informática na Educação (CBIE 2019)

Anais do XXX Simpósio Brasileiro de Informática na Educação (SBIE 2019)

BRASIL. Instituto Nacional de Estudos e Pesquisas Educacionais Anísio Teixeira. "Censo Escolar 2016 - Notas Estatísticas". Brasília, 2016. Disponível em: <http://download.inep.gov.br/educacao_basica/censo_escolar/notas_estatisticas/2017 /notas_estatisticas_censo_escolar_da_educacao_basica_2016.pdf>. Acesso em: julho de 2018.

BROOKE, J.; SUS: A 'Quick and Dirty' usability scale. In: JORDAN, P.W. et al. (Orgs.). Usability Evaluation in Industry. London: Taylor \&amp; Francis, 1996. cap. 21, p. $189-194$.

CAMPOS, B. O. "Utilização de simulações computacionais no ensino de física, na área da termologia". 2017. Dissertação (Mestrado em Ensino de Física) - Universidade Federal de Alfenas, Alfenas - MG.

CARMO, R. O. “Geogebra no Ensino de Física: Propostas de Aplicação para o Ensino do Movimento Harmônico Simples”. 2017. Dissertação (Mestrado Profissional em Ensino de Física) - Universidade Federal de São Carlos -campus Sorocaba, Sorocaba.

ESTEVÃO, Isabel Cristina Alves. "WebQui - uma plataforma virtual para ensinar Química de forma interativa, dinâmica e visual". 2019. 103 f. Dissertação (Mestrado em Ensino de Ciências) - Instituto de Ciências Exatas e Biológicas, Universidade Federal de Ouro Preto, Ouro Preto, 2019.

OLIVEIRA, Humberto da Silva. "Uma investigação da modelagem e simulação computacional no Ensino de Física". 2014. 136 p. Dissertação (Mestrado Profissional em Ensino de Ciências e Matemática) - Universidade Estadual da Paraíba, Centro de Ciências e Tecnologia, 2014.

ROSSI, Diego Delovo. "O uso de simulações virtuais como apoio ao currículo da SEESP para a disciplina de Física". 2015. xi, 33 f. Dissertação (mestrado) - Universidade Estadual Paulista, Faculdade de Ciências e Tecnologia, 2015.

TOULMIN, S.; The uses of argument, University Press: Cambridge, 1958

VIGOTSKY, LEV S. "A formação social da mente: o desenvolvimento dos processos psicológicos superiores". $3^{\mathrm{a}}$.ed. São Paulo: Martins Fontes, 1989. 168p. (Coleção Psicologia e Pedagogia. Nova Série)

WILSEK, M.; TOSIN, J. "Ensinar e aprender ciências no ensino fundamental com atividades investigativas através da resolução de problemas”. Estado do Paraná, v. 3, n. 5, 2012. 